\title{
Effect of Doping Concentration on Conversion Efficiency of Silicon Based Nano-gap Near-field Thermophotovoltaic Cells
}

\author{
Japheth Z-J. Lau* and Basil T. Wong \\ Swinburne Sarawak Research Centre for Sustainable Technologies, \\ Faculty of Engineering, Computing and Science, \\ Swinburne University of Technology Sarawak Campus, \\ Jalan Simpang Tiga, 93350 Kuching, Sarawak, Malaysia \\ *Corresponding author: jzjlau@swinburne.edu.my
}

Published online: 25 November 2018

To cite this article: Lau, J. Z-J. \& Wong, B. T. (2018). Effect of doping concentration on conversion efficiency of silicon based nano-gap near-field thermophotovoltaic cells. J. Phys. Sci., 29(3), 37-54, https://doi.org/10.21315/jps2018.29.3.4

To link to this article: https://doi.org/10.21315/jps2018.29.3.4

\begin{abstract}
We studied the doping dependence of a nano-gap thermophotovoltaic device that utilises a p-on- $n$ Si photovoltaic cell. The concentration of acceptor dopants, $N_{a}$ in the emitter region was varied from $10^{23} \mathrm{~m}^{-3}$ to $10^{25} \mathrm{~m}^{-3}$ while the donor concentration, $N_{d}$ in the absorber region was varied from $10^{21} \mathrm{~m}^{-3}$ to $10^{24} \mathrm{~m}^{-3}$. The combination of $N_{a}$ and $N_{d}$ that produces the highest conversion efficiency is $N_{a}=10^{23} \mathrm{~m}^{-3}$ and $N_{d}=10^{22} \mathrm{~m}^{-3}$. However, the absorber doping concentration that produces the greatest output power is around $10^{23} \mathrm{~m}^{-3}$ at all $N_{a}$ values. It is discovered that at higher $N_{a}$ values, the optical response of the device is less sensitive to $N_{d}$. It is shown that a decreasing diffusion length does not necessarily jeopardise performance especially when it already exceeds the absorber region thickness. This is due to the positive effects of a decreasing diffusion coefficient.
\end{abstract}

Keywords: Doping-dependence, nano-gap TPV, near-field heat transfer, silicon cell, thermophotovoltaics

\section{INTRODUCTION}

A nano-gap thermophotovoltaic (TPV) device is an energy generator that converts heat into electricity. The separation distance between the radiator and the TPV cell (receiver) is nanometric in size, hence the name. Just like conventional solar cells, it utilises photovoltaic (PV) cells to convert electromagnetic waves into electricity. When referring to TPV energy conversion, the term TPV cell is used to differentiate it from its solar-powered counterpart. 
A basic PV cell design involves doping one section of an intrinsic material with acceptor dopants ( $p$-type) and the other section with donor dopants ( $n$-type) to form a $p-n$ junction. Many studies have shown that varying the dopant concentration affects the performance of PV cells. One such study was conducted by Durán et al. in which they attempted to optimise the junction depth and doping concentration of solar cell emitters. ${ }^{1}$ The term "emitter" here refers to the top layer of the $p-n$ junction and should not be confused with the TPV radiation source. They varied the emitter doping concentration from $8 \times 10^{18} \mathrm{~cm}^{-3}$ to $10^{20} \mathrm{~cm}^{-3}$ and discovered that the cell efficiency increased with doping concentration up to an optimum value of $1.5 \times 10^{19} \mathrm{~cm}^{-3}$ above which the efficiency decreased. Karazhanov studied the doping-dependence of a silicon, Si solar cell by varying the doping concentration of its $p$-type base region. ${ }^{2}$ As doping concentration is increased from $10^{16} \mathrm{~cm}^{-3}$ to $10^{18} \mathrm{~cm}^{-3}$, the minority carrier diffusion length, short-circuit current $\left(J_{s c}\right)$, opencircuit voltage $\left(V_{o c}\right)$, fill factor $(F F)$ and efficiency experience declined. They concluded that the optimal doping concentration should be less than $10^{17} \mathrm{~cm}^{-3}$. Relatively similar conclusions were made by Ouyang et al. in their experimental study of thin film polycrystalline $\mathrm{Si}$ solar cells. ${ }^{3}$ The doping concentration of the $p$-type base/absorber region was varied from around $2 \times 10^{15} \mathrm{~cm}^{-3}$ to $8 \times 10^{17} \mathrm{~cm}^{-3}$. Their results show that $J_{s c}$ values are greatest at lower doping concentrations while $V_{o c}$ and pseudo fill factor values peak at concentrations of $1-2 \times 10^{17} \mathrm{~cm}^{-3}$. They noted that $J_{s c}$ is the dominating determinant of conversion efficiency, thus cell efficiency decreases as base doping concentration increases. Furthermore, they determined that the effective diffusion length $\left(L_{e f f}\right)$ of minority carriers is one the major parameters that affect quantum efficiency. A high $L_{\text {eff }}$ would increase the collection probability of light-generated carriers, subsequently increasing efficiency. It has to be noted that these studies use an $n$-on- $p$ cell configuration where the $n$-type layer is placed above the $p$-type layer with the $n$-type layer receiving light first.

The doping concentration of a semiconductor also influences near-field heat transfer. Fu and Zhang studied the thermal energy transfer between Si parallel plates at different doping concentrations. ${ }^{4}$ Based on their calculations, an increase in doping concentration significantly increases the extinction coefficient of Si due to increased free carrier absorption. This increased contribution from free carriers causes greater spectral radiative heat flux at lower angular frequencies (below $10^{14} \mathrm{rad} \mathrm{s}^{-1}$ ). Moreover, the net energy flux between two heavily doped Si plates is significantly higher than Si plates with lower doping concentrations. In another paper, the PV cell properties such as minority carrier mobility, diffusion coefficient, lifetime and depletion region length are all shown to be doping-dependent. ${ }^{5}$ 
These studies allow us to safely conclude that changing the doping concentration of the TPV cell in a nano-gap TPV device would definitely affect its optical and electrical responses which in turn determine the performance of the entire system. Through this paper, we would like to shed more light on the relationship between doping concentration and the aforementioned responses of the device. It is hoped that this insight may allow researchers to make more informed decisions when conducting experimental studies on nano-gap TPV devices. The dopingdependence of a nano-gap TPV device that utilises a $p$-on- $n$ Si TPV cell will be studied. A $p$-on- $n$ configuration is chosen to negate the possibility of a short circuit at the cell junction when a top ohmic contact is annealed to an $n$-type material. ${ }^{6}$ The radiator is kept at $2000 \mathrm{~K}$ in order to produce a dominant wavelength of $1.5 \mu \mathrm{m}$ (guaranteeing that any nanometric gap is within the near-field regime) while the TPV cell remains at $300 \mathrm{~K}$ (close to room temperature) with an arbitrarily chosen gap of $10 \mathrm{~nm}$ separating the two layers. We believe larger gaps would produce the same trends as long as the near-field regime is induced (the gap is smaller than the dominant wavelength of radiation). It has to be noted that the configuration used here is based on the work of Park et al. ${ }^{7}$

\section{MODELLING AND SIMULATION}
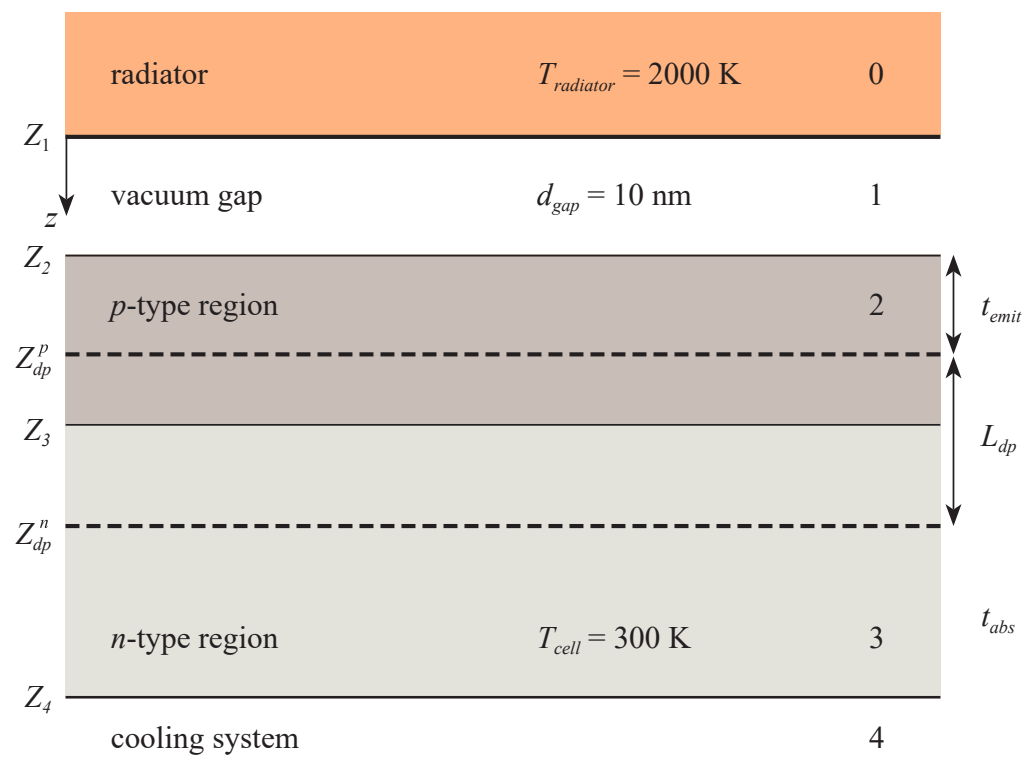

Figure 1: Schematic diagram of a nano-gap TPV device. 
The nano-gap TPV device depicted in Figure 1 is simulated using the algorithm and simulation parameters described in Francoeur et al. ${ }^{8}$ Silicon carbide (SiC, a common infrared radiator) is used as the radiator (layer 0 ) while a $\mathrm{Si}$ (a common PV cell material) $p$ - $n$ junction (layers 2 and 3 ) is used as the TPV cell. Media 1 and 4 are modelled as vacuum. The one-dimensional near-field heat transfer problem is solved using fluctuational electrodynamics where the Maxwell equations are solved using the fluctuation-dissipation theorem. The position-dependent spectral radiative heat flux is calculated using Equation 1 where $l$ is the absorbing layer where the point of interest, $z_{c}$ is located. The analytical expression - in terms of the reflection coefficients - of the incident spectral heat flux transferred from a bulk radiator to an absorbing $p-n$ junction is also derived and is calculated as the sum of Equations 2 and 3., ${ }^{9}$ The reflection coefficients of layers 2 and 3 are written as Equations 4 and 5, respectively. The interfacial Fresnel reflection coefficients are taken from Yeh. ${ }^{11}$ It has to be noted that the Fresnel transmission coefficients are negligible and so are not taken into account. The term $\Theta$ is the mean energy of a Planck oscillator.

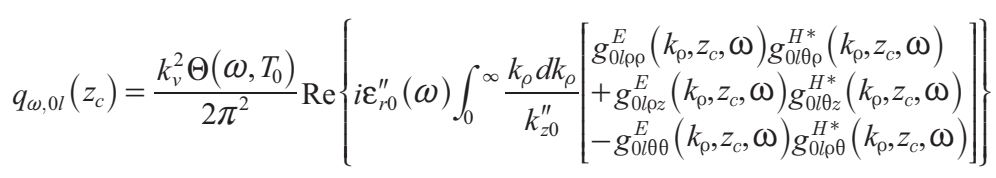

$$
\begin{aligned}
& q_{\omega}^{\text {prop }}\left(z_{2}^{+}\right)=\frac{\Theta\left(\omega, T_{0}\right)}{4 \pi^{2}} \int_{0}^{k_{y}} k_{\rho} d k_{\rho} \sum_{\gamma=T E, T M} \frac{\left(1-\left|r_{10}^{\gamma}\right|^{2}\right)\left(1-\left|R_{2}^{\gamma}\right|^{2}\right)}{\mid 1-r_{10}^{\gamma} R_{2}^{\gamma} e^{\left.2 i k_{11}^{\prime} d_{g u}\right|^{2}}} \\
& q_{\omega}^{\text {evan }}\left(z_{2}^{+}\right)=\frac{\Theta\left(\omega, T_{0}\right)}{\pi^{2}} \int_{k_{v}}^{\infty} k_{\rho} d k_{\rho} e^{-2 k_{z 1}^{\prime \prime} d_{\text {gep }}} \sum_{\gamma=T E, T M} \frac{\operatorname{Im}\left(r_{10}^{\gamma}\right) \operatorname{Im}\left(R_{2}^{\gamma}\right)}{\left|1-r_{10}^{\gamma} R_{2}^{\gamma} e^{2 i k_{11}^{\prime \prime} d_{g e p}}\right|^{2}} \\
& R_{2}^{\gamma}=\frac{r_{1,2}^{\gamma}+R_{3}^{\gamma} e^{2 i k_{22} t_{2}}}{1+r_{1,2}^{\gamma} R_{3}^{\gamma} e^{2 i k_{22} t_{2}}} \\
& R_{3}^{\gamma}=\frac{r_{2,3}^{\gamma}+r_{3,4}^{\gamma} e^{2 i k_{3} t_{3}}}{1+r_{2,3}^{\gamma} r_{3,4}^{\gamma} e^{2 i k_{33} t_{3}}}
\end{aligned}
$$

The TPV cell operation is modelled based on the work done in Vaillon et al. where the minority carrier transport is described by the minority carrier diffusion equation (Equation 6) which is used to determine the minority carrier densities, $\Delta n .^{5,12}$

$$
D_{(e, h)} \frac{d^{2} \Delta n_{(e, h), \omega}(z)}{d z^{2}}-\frac{\Delta n_{(e, h), \omega}(z)}{\tau_{(e, h)}}+g_{\omega}(z)=0
$$




$$
\begin{aligned}
& D_{(e, h)} \frac{d \Delta n_{(e, h), \omega}\left(Z_{2,4}\right)}{d z}=S_{(e, h)} \Delta n_{(e, h), \omega}\left(Z_{2,4}\right) \\
& \Delta n_{(e, h), \omega}\left(Z_{d p}^{(p, h)}\right)=0
\end{aligned}
$$

Equation 6 is solved using the Thomas algorithm with the inclusion of boundary conditions as described by Equations 7 and $8 .{ }^{13}$ The model uses the depletion region approximation which assumes that the depletion region is completely depleted of minority carriers and the regions on either side are quasi-neutral. Thus, the photocurrents can be calculated using Equations 9 to 11. Summing them up and integrating over all frequency values would yield the total photocurrent generated, $J_{p h}$.

$$
\begin{aligned}
& J_{d p, \omega}=e \int_{Z_{d p}^{p}}^{Z_{d p}^{n}} g_{j, \omega} d z \\
& J_{e, \omega}=e D_{e} \frac{d \Delta n_{e, \omega}\left(Z_{d p}^{p}\right)}{d z} \\
& J_{h, \omega}=-e D_{h} \frac{d \Delta n_{h, \omega}\left(Z_{d p}^{n}\right)}{d z} \\
& \Delta n_{(e, h), \omega} Z_{d p}^{(p n)}=n_{(e, h) 0} \exp \left(\frac{e V_{f}}{k_{b} T_{c e l l}}\right)
\end{aligned}
$$

Using this model, the $J-V$ characteristic is generated by solving for the net current $J\left(V_{f}\right)=J_{p h}-J_{0}\left(V_{f}\right)$ at increasing values of the forward bias, $V_{f}$ and from there the maximum output power, $P_{m}$ can be obtained. The dark current, $J_{0}$ is obtained by solving the minority carrier diffusion equation in dark conditions, i.e., $g_{\omega}=0$. The boundary condition at the edges of the depletion region in the dark is described by Equation $12 .^{5}$ The $J_{s c}$ and $V_{o c}$ of the TPV cell are also obtained from the $J-V$ characteristic. The efficiency of the nano-gap TPV device is calculated using Equation 13 where $P_{a b s}$ is the total radiative heat flux absorbed by the TPV cell. These quantities are used to evaluate the performance of the whole system.

$$
\eta_{c}=100 \times \frac{P_{m}}{P_{a b s}}
$$

The SiC radiator is modelled as a damped harmonic oscillator (Equation 14) using the parameters (Table 1) found in Palik. ${ }^{14}$ The Si TPV cell's dielectric function is described using a Drude model (Equation 15) detailed in Fu and Zhang with the addition of a lattice absorption model from Collins and Fan. ${ }^{4,15}$ The ionisation model of dopants used in our work is taken from Kuzmicz. ${ }^{16}$ 
Table 1: Parameters for modelling SiC.

\begin{tabular}{cccc}
\hline$\varepsilon_{\infty}$ & $\omega_{T O}\left(\mathrm{rad} \mathrm{s}^{-1}\right)$ & $\omega_{L O}\left(\mathrm{rad} \mathrm{s}^{-1}\right)$ & $\Gamma\left(\mathrm{s}^{-1)}\right.$ \\
\hline 6.7 & $1.494 \times 10^{14}$ & $1.825 \times 10^{14}$ & $8.966 \times 10^{11}$ \\
\hline$\varepsilon_{r}(\omega)=\varepsilon_{\infty}\left(\frac{\omega^{2}-\omega_{L O}^{2}+i \Gamma \omega}{\omega^{2}-\omega_{T O}^{2}+i \Gamma \omega}\right)$ \\
$\varepsilon(\omega)=\varepsilon_{b l}-\frac{N_{e} e^{2} / \varepsilon_{0} m_{e}^{*}}{\omega^{2}-i \omega / \tau_{e}}-\frac{N_{h} e^{2} / \varepsilon_{0} m_{h}^{*}}{\omega^{2}-i \omega / \tau_{h}}$
\end{tabular}

The SiC radiator is kept at $2000 \mathrm{~K}$ while the TPV cell is kept at $300 \mathrm{~K}$. The frequency range of the simulation starts from $5 \times 10^{12} \mathrm{rad} \mathrm{s}^{-1}$ to $3.8 \times 10^{15} \mathrm{rad} \mathrm{s}^{-1}$. The thickness of the $p$-type region is $0.4 \mu \mathrm{m}$ while the $n$-type region is $10 \mu \mathrm{m}$ thick as done in Park et al. ${ }^{7}$ It is unclear why Park et al. chose those thicknesses; it could be that those thicknesses were arbitrarily chosen as long as they were larger than the depletion region width. The diffusion lengths of minority electrons ( $p$-type) and holes (n-type) could be another reason. Electrons have shorter diffusion lengths compared to holes. Furthermore, layers which are too thick would reduce the collection probability of those minority carriers. The surface recombination velocities of the TPV cell are taken from Vaillon et al. where $S_{e}=10 \mathrm{~m} \mathrm{~s}^{-1}$ and $S_{h}=1 \mathrm{~m} \mathrm{~s}^{-1}$. $^{5}$

\section{RESULTS AND DISCUSSIONS}

The doping concentration in the $p$-type region (acceptor), $N_{a}$ is varied from $10^{23} \mathrm{~m}^{-3}$ to $10^{25} \mathrm{~m}^{-3}$. For each $N_{a}$ value, the $n$-type region doping concentration (donor), $N_{d}$ is varied from $10^{21} \mathrm{~m}^{-3}$ to $10^{24} \mathrm{~m}^{-3}$. These values are chosen based on the doping concentrations used in past studies which are mentioned in the introduction. The results are presented in the following discussion. 


\subsection{Performance}

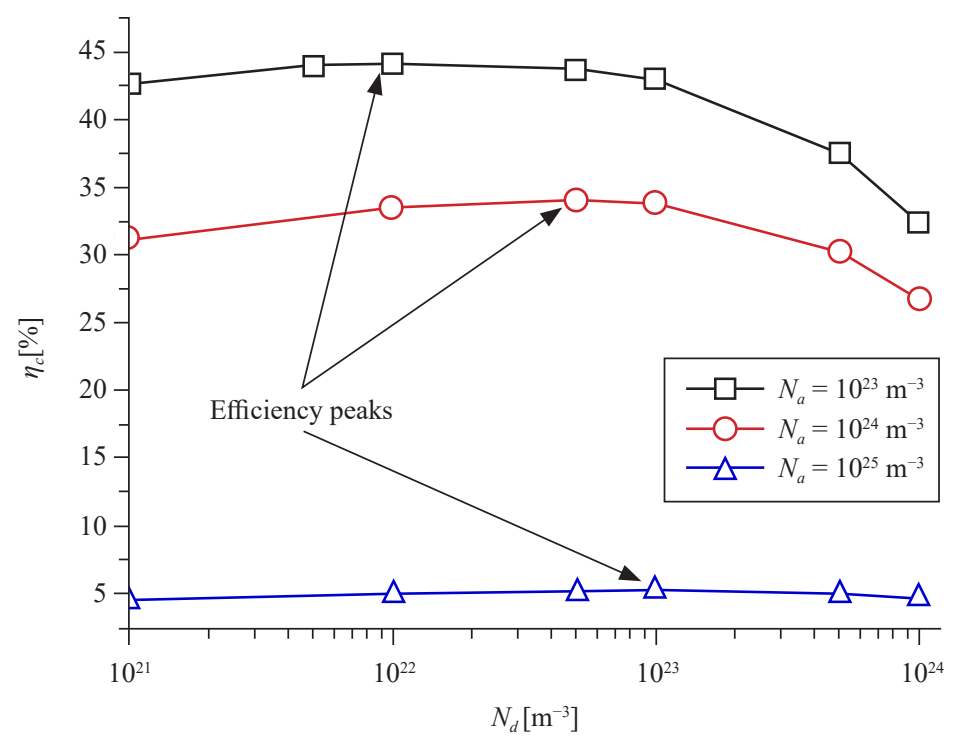

Figure 2: Conversion efficiencies at different doping concentrations (note that only symbols represent actual data points. This is true for all figures with symbols).

As shown in Figure 2, the highest conversion efficiency among the simulated conditions occurs when $N_{a}=10^{23} \mathrm{~m}^{-3}$ and $N_{d}=10^{22} \mathrm{~m}^{-3}$. It is interesting to note that as $N_{a}$ is increased, the optimum $N_{d}$ for that particular $N_{a}$ value increases too up to $N_{d}=10^{23} \mathrm{~m}^{-3}$ where $P_{m}$ is greatest, shown in Figure 3(b). This is because at higher $N_{a}$ values, the rise in $P_{a b s}$ in Figure 3(a) as $N_{d}$ is increased dwindles causing the greatest efficiency to approach the point where $P_{m}$ is greatest. Referring to Figure $4, J_{s c}$ and $V_{o c}$ are maximum at around $N_{d}=10^{23} \mathrm{~m}^{-3}$ which coincides with the point of maximum $P_{m}$. It is commonly reported that the doping concentration of the TPV cell base region needs to be as low as possible in order to maximise the effective minority carrier diffusion length, $L_{e f f}$ in order to achieve greater $J_{s c}$ values which should improve efficiency. ${ }^{3}$ However, the diffusion length may not be the only deciding factor, especially when TPV cells are relatively thin as is the case in this paper. The results shown in Figures 2 to 4 do seem to indicate that increasing $N_{d}$ does improve performance up to a certain optimum value. This interesting observation will be analysed in Section 3.3. 


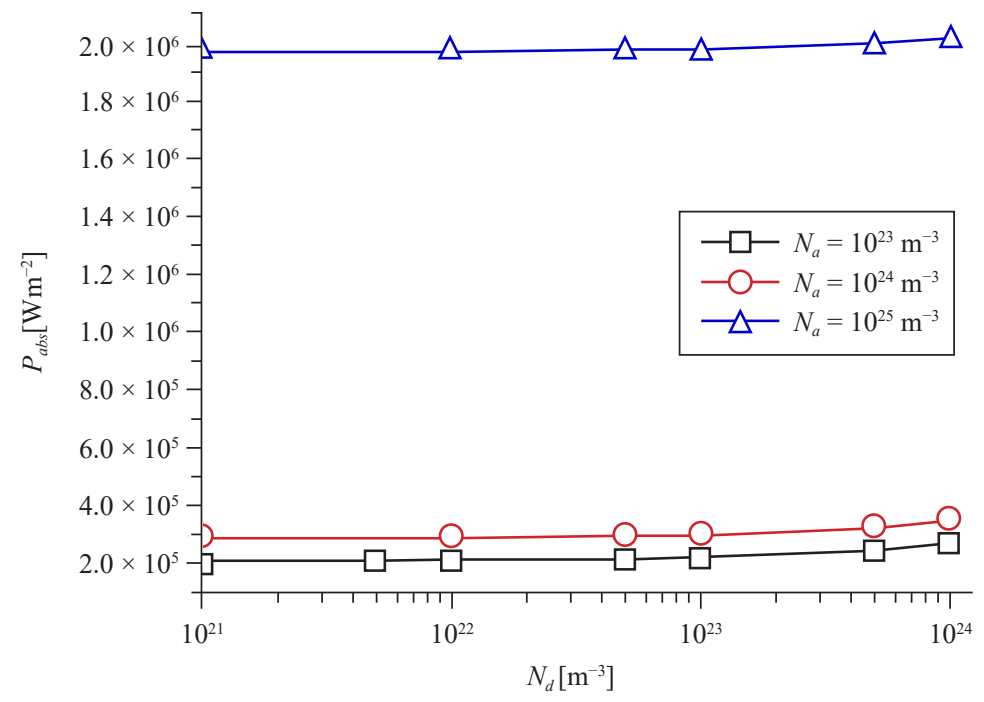

(a)

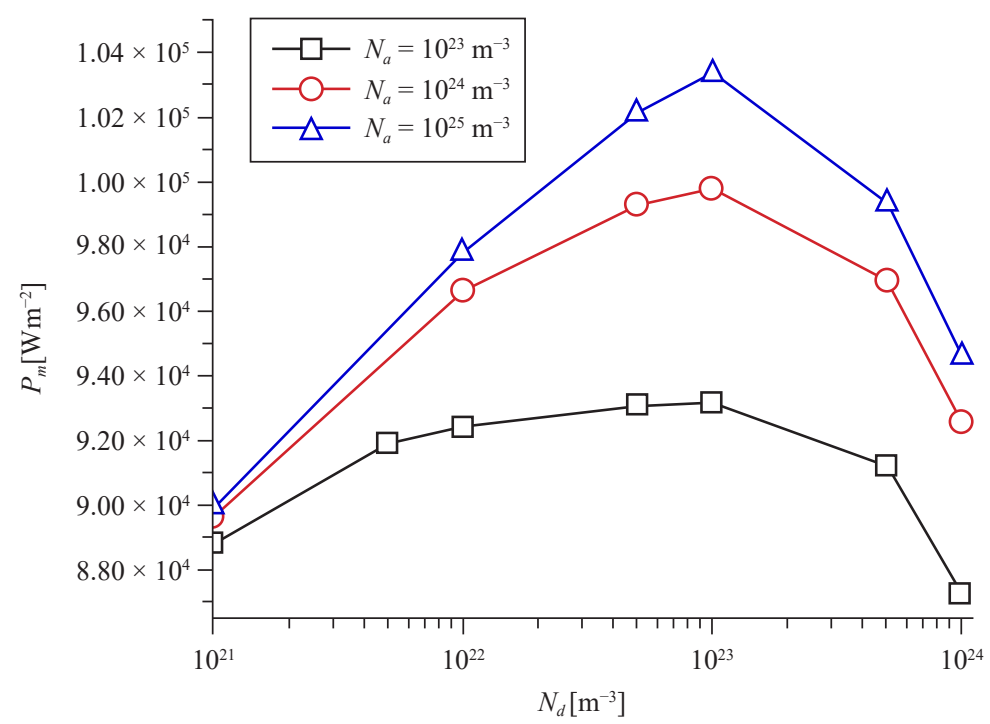

(b)

Figure 3: Plots of (a) total absorbed radiative heat flux and (b) maximum output power at different doping concentrations. 


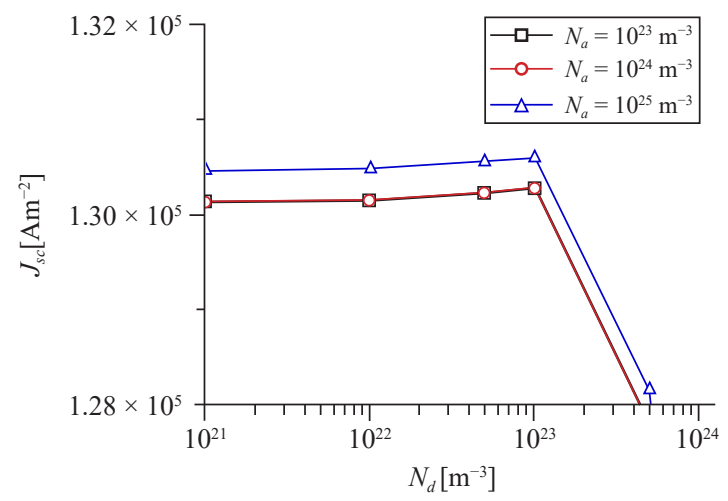

(a)

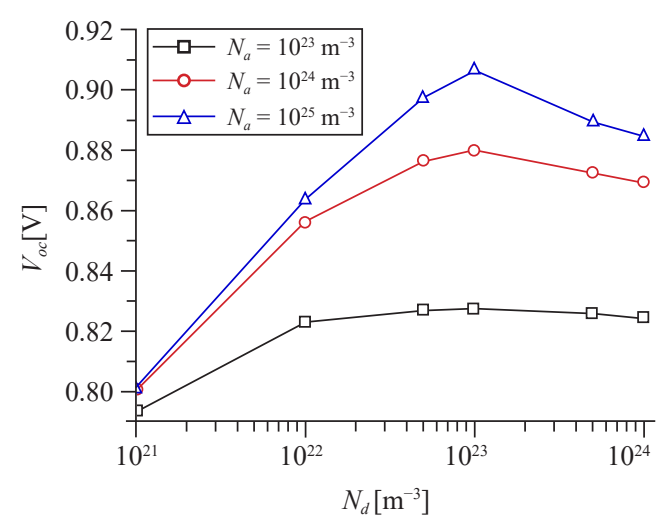

(b)

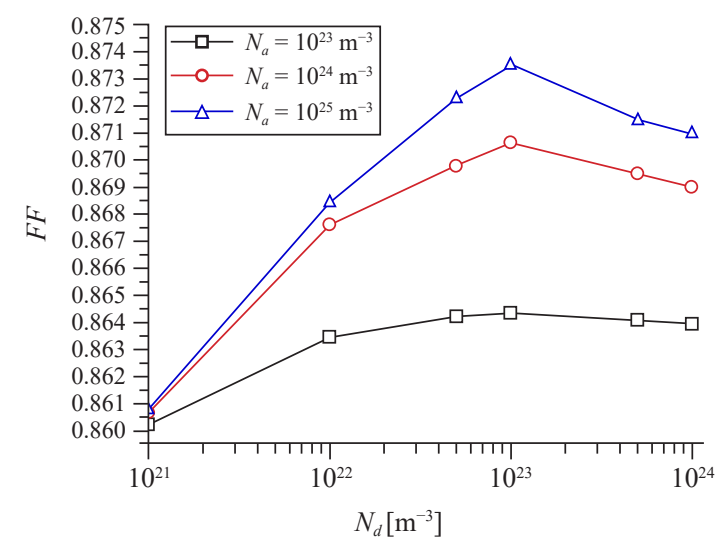

(c)

Figure 4: Plots of (a) short-circuit current, (b) open-circuit voltage and (c) fill factor at different doping concentrations. 


\subsection{Optical Response}

In this section, the optical response of the nano-gap TPV device is analysed. The effect of doping concentration on the spectral radiative heat flux profile is presented.

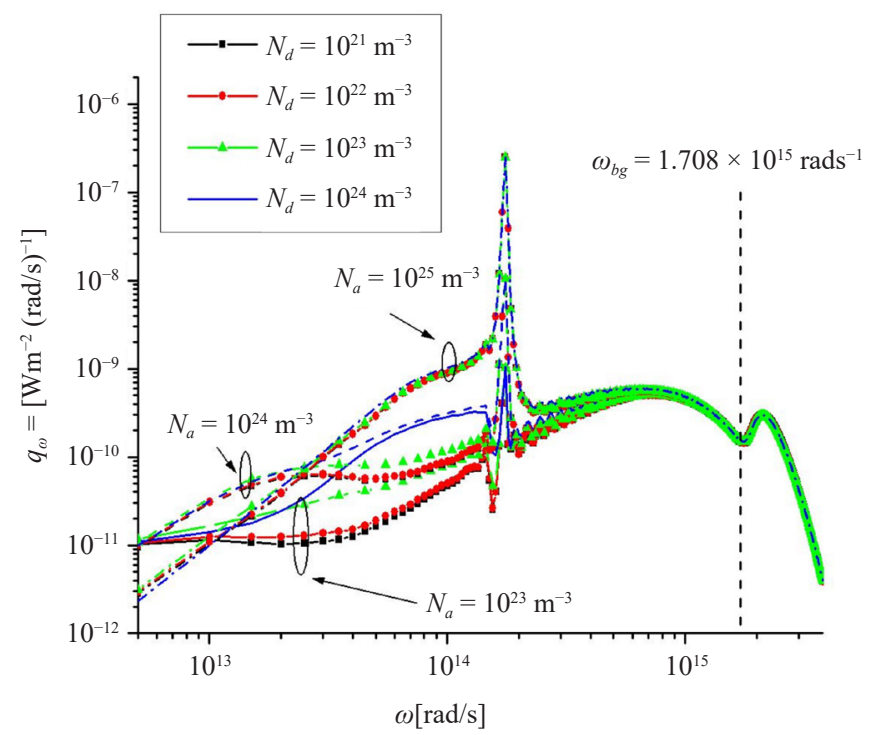

(a)

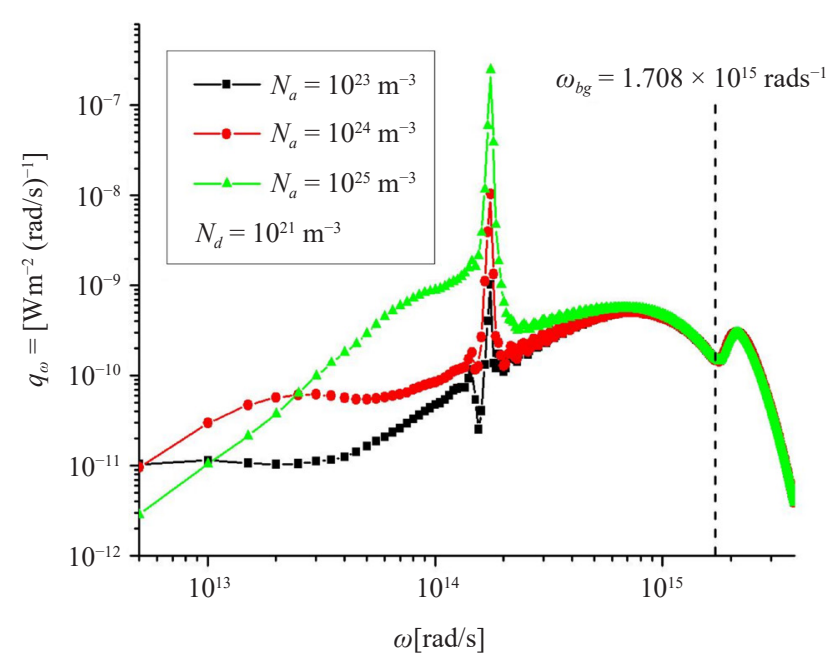

(b)

Figure 5: Monochromatic incident radiative heat flux as a function of angular frequency for (a) different combinations of $N_{a}$ and $N_{d}$ and (b) when $N_{d}$ is fixed at $10^{21} \mathrm{~m}^{-3}$. 
Based on Figure 5(b), as $N_{a}$ is increased, the incident spectral radiative heat flux increases significantly below the band gap frequency, $\omega_{b g}$ while above band gapimprovements are rather small. The narrow heat flux peak is caused by surface phonon-polariton resonance. The increase in heat flux can be explained by the greater absorption coefficient in highly doped silicon due to increased free carrier absorption. ${ }^{4}$ Greater absorption increases the so-called spectral absorptance (generalised emissivity), $\left(1-\left|R_{2}^{\gamma}\right|^{2}\right)$ and $\operatorname{Im}\left(R_{2}^{\gamma}\right)$ (in Equations 2 and 3) of the TPV cell. ${ }^{17-19}$ However, greater absorption below the band gap does not result in increased generation rate, thus the conversion efficiency of the nano-gap TPV device drops drastically as $N_{a}$ increases.

Referring to Figure 5(a), at lower $N_{a}$ values, as $N_{d}$ increases, the heat flux sees noticeable increase between $10^{13} \mathrm{rad} \mathrm{s}^{-1}$ and $1.5 \times 10^{14} \mathrm{rad} \mathrm{s}^{-1}$ due to increased free carrier absorption. However, at $N_{a}=10^{25} \mathrm{~m}^{-3}$, the change in heat flux induced by the change in $N_{d}$ becomes insignificant which explains the weaker rise in $P_{a b s}$. This shows that at higher absorption coefficients, the optical response of the surface layer is the dominant factor in determining the incident heat flux. Thus, in order to achieve maximum radiative heat transfer, only a very thin layer at the surface of the TPV cell needs to be highly doped. It is interesting to note that even though the increase in $N_{d}$ generates more unusable spectral heat flux, the conversion efficiency actually increases up to a certain optimum value which differs for different values of $N_{a}$. This shows that the improvements brought about by increasing $N_{d}$ outweighs its negative effects.

\subsection{Electrical Response}

In this section, the effect of doping concentration on the electrical properties of the TPV cell and subsequently the generated photocurrent, $J_{p h}$ at short-circuit conditions is analysed and the reason for the improvements generated by increasing $N_{d}$ is explained. 


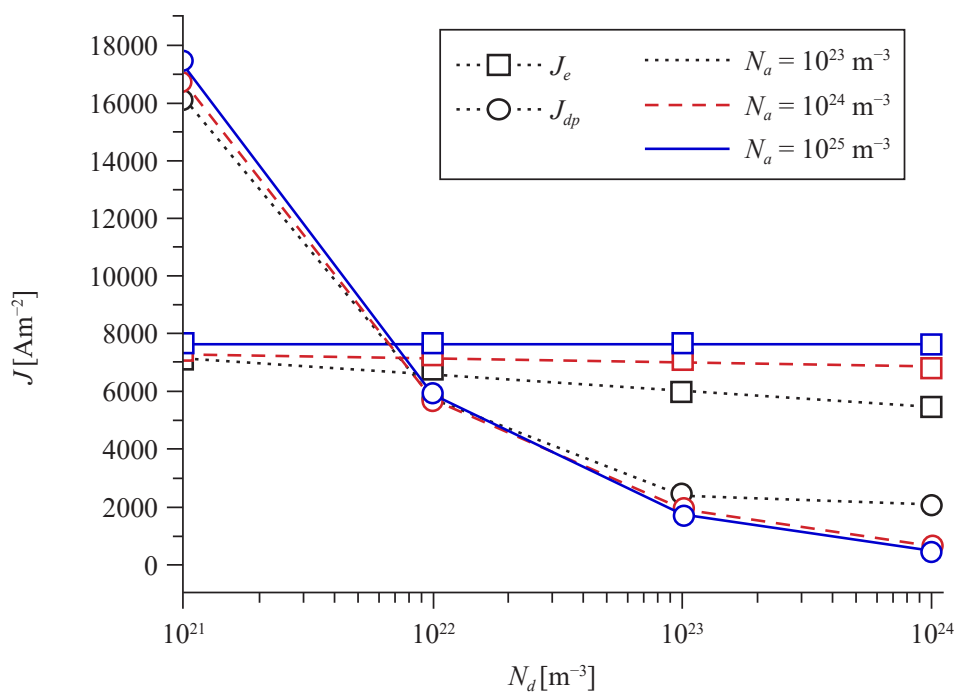

(a)

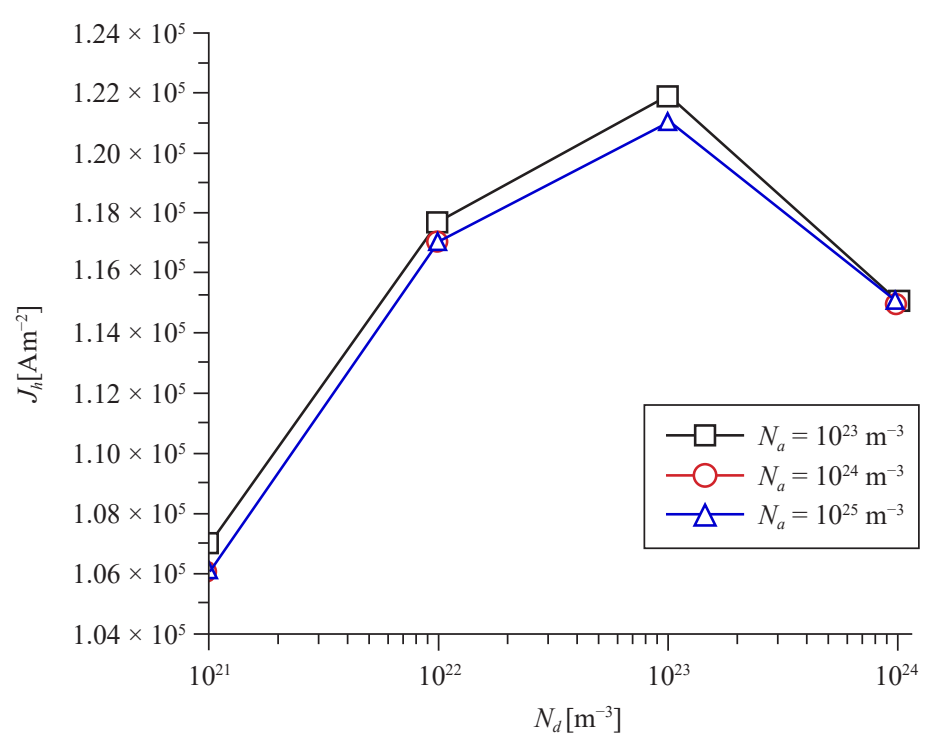

(b)

Figure 6: Photocurrents of (a) $J_{e}, J_{d p}$ and (b) $J_{h}$ at different doping concentrations. 


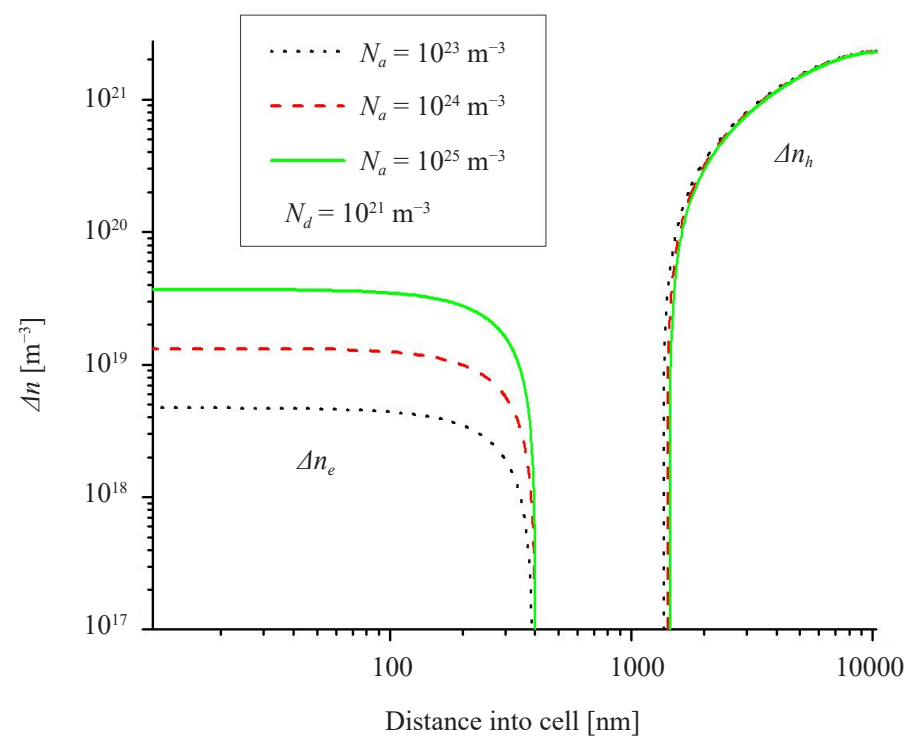

(a)

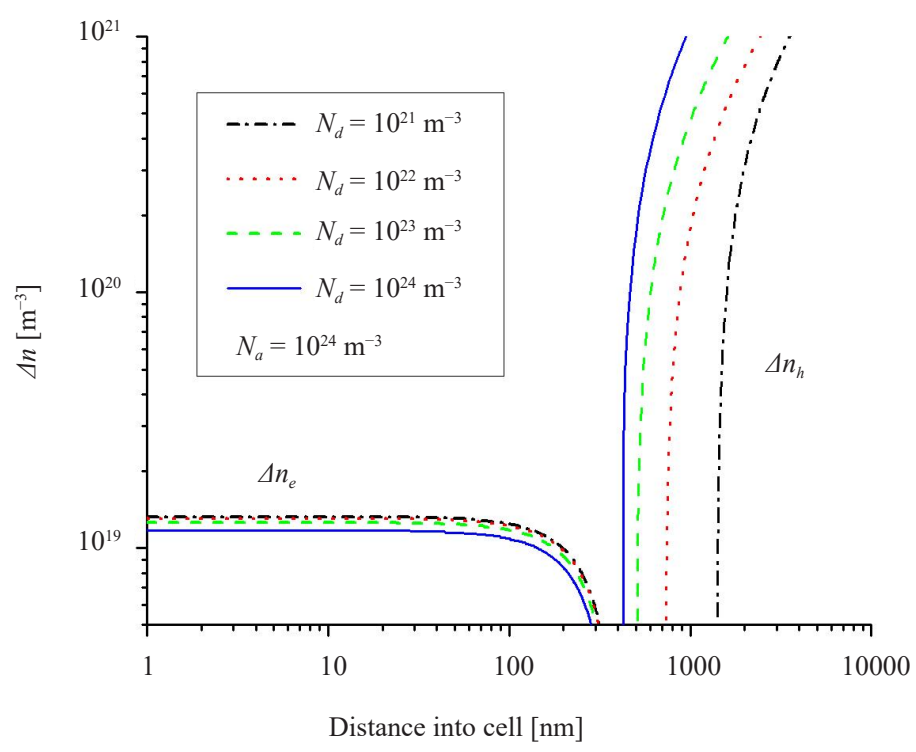

(b)

Figure 7: Excess minority carrier concentration as a function of distance into cell when (a) $N_{d}$ is fixed at $10^{21} \mathrm{~m}^{-3}$ and (b) $N_{a}$ is fixed at $10^{24} \mathrm{~m}^{-3}$. 
Table 2: Diffusion coefficient, lifetime and effective diffusion length of minority electrons at different acceptor concentrations.

\begin{tabular}{cccc}
\hline$N_{a}\left(\mathrm{~m}^{-3}\right)$ & $D_{e}\left(\mathrm{~m}^{2} \mathrm{~s}^{-1}\right)$ & $\tau_{e}(\mathrm{~s})$ & $L_{\mathrm{eff}, e}(\mathrm{~m})$ \\
\hline $10^{23}$ & $1.86 \times 10^{-3}$ & $2.89 \times 10^{-6}$ & $7.33 \times 10^{-5}$ \\
$10^{24}$ & $6.91 \times 10^{-4}$ & $2.82 \times 10^{-7}$ & $1.40 \times 10^{-5}$ \\
$10^{25}$ & $2.61 \times 10^{-4}$ & $2.27 \times 10^{-8}$ & $2.43 \times 10^{-6}$ \\
\hline
\end{tabular}

Table 3: Diffusion coefficient, lifetime and effective diffusion length of minority holes at different donor concentrations.

\begin{tabular}{cccc}
\hline$N_{d}\left(\mathrm{~m}^{-3}\right)$ & $D_{h}\left(\mathrm{~m}^{2} \mathrm{~s}^{-1}\right)$ & $\tau_{h}(\mathrm{~s})$ & $L_{\text {eff }, h}(\mathrm{~m})$ \\
\hline $10^{21}$ & $1.21 \times 10^{-3}$ & $1.25 \times 10^{-5}$ & $1.23 \times 10^{-4}$ \\
$10^{22}$ & $1.10 \times 10^{-3}$ & $1.25 \times 10^{-5}$ & $1.17 \times 10^{-4}$ \\
$10^{23}$ & $7.76 \times 10^{-4}$ & $1.25 \times 10^{-5}$ & $9.85 \times 10^{-5}$ \\
$10^{24}$ & $3.70 \times 10^{-4}$ & $1.04 \times 10^{-6}$ & $1.96 \times 10^{-5}$ \\
\hline
\end{tabular}

\subsubsection{Varying $\mathbf{N}_{\mathrm{a}}$}

Referring to Figure 6 , as $N_{a}$ increases, $J_{e}$ experiences improvements while $J_{h}$ declines. The increase in $J_{e}$ is due to the build-up of excess minority carriers, $\Delta n_{e}$ shown in Figure 7(a) in the emitter region. This build-up is caused by greater electron-hole pair (EHP) generation as $t_{\text {emit }}$ increases and the diffusion coefficient decreases (Table 2). The value of $J_{d p}$ depends on the wideness of $L_{d p}$ which is the sum of the depletion region width in both $p$ and $n$-type regions. Furthermore, they are all functions of $N_{a}$ and $N_{d}{ }^{12,20}$ Thus, a wider depletion region width generates more $J_{d p}$. When $N_{d}$ is below $10^{22} \mathrm{~m}^{-3}$, an increasing $N_{a}$ widens $L_{d p}$. Above $N_{d}=10^{22} \mathrm{~m}^{-3}$, the opposite is true as the width becomes narrower which explains the decreasing $J_{d p}$ in Figure 6(a). However, the depletion region width in the $n$-type region continues to increase ( $t_{a b s}$ decreases), causing less radiative heat flux to be absorbed in the absorber region thus generating less EHPs which reduces $J_{h}$ albeit in small amounts. Overall, the increase in $J_{e}$ and $J_{\mathrm{dp}}$ causes $J_{p h}$ to increase as $N_{a}$ is increased from $10^{23} \mathrm{~m}^{-3}$ to $10^{25} \mathrm{~m}^{-3}$.

\subsubsection{Varying $\mathbf{N}_{\mathrm{d}}$}

Referring to Figure $6, J_{e}$ and $J_{d p}$ decrease as $N_{d}$ is increased. Greater $N_{d}$ values cause the depletion region width in the $p$-type region to expand when $N_{a}$ is fixed. This reduces the thickness of the TPV cell emitter region, $t_{\text {emit }}$ which subsequently reduces the amount of EHPs generated in this region, hence the declining $J_{e}$. However, the overall width, $L_{d p}$ shrinks causing less $J_{d p}$ to be generated. 
$J_{h}$ increases as $N_{d}$ is increased from $10^{21} \mathrm{~m}^{-3}$ to $10^{23} \mathrm{~m}^{-3}$ but drops when $N_{d}$ is increased further. $J_{h}$ is proportional to $D_{h}$ and also the excess minority carrier gradient at the depletion region boundary in the $n$-type region. ${ }^{5}$ The increase of $J_{h}$ as doping concentration increases can be explained by the increase in the gradient due to the buildup of $\Delta n_{h}$ (Figure 7(b)) during illumination as a result of the lower $D_{h}$ (Table 3 ) while $\tau_{h}$ remains largely unchanged, i.e., the recombination rate is hardly affected. This shows that a lower $D$ is only useful when $\tau$ remains relatively constant. However, it has to be noted that $L_{\text {eff; } h}$ continues to decrease. At $10^{24} \mathrm{~m}^{-3}$, the effect of a much lower $D_{h}$ on $J_{h}$ outweighs the buildup of $\Delta n_{h}$, hence the smaller $J_{h}$ value. Furthermore, at $10^{24} \mathrm{~m}^{-3}, \tau_{h}$ experiences quite a significant decline which also compromises the buildup of $\Delta n_{h}$. This ties in with the physical picture where a lower $D_{h}$ and $\tau_{h}$ reduce the collection probability of EHPs, causing less current to be generated. However, it is clear that relying on $L_{\text {eff }}$ as a measure to predict performance is not sufficient especially when the TPV cell is thin to begin with. It would be wiser to include in the analysis the effects of $D$ and $\tau$ as the interplay between these two parameters paints a more complete picture. $J_{h}$ is the main contributor of photocurrent and thus the $J_{p h}$ profile matches the $J_{h}$ profile.

\subsubsection{Dark current}

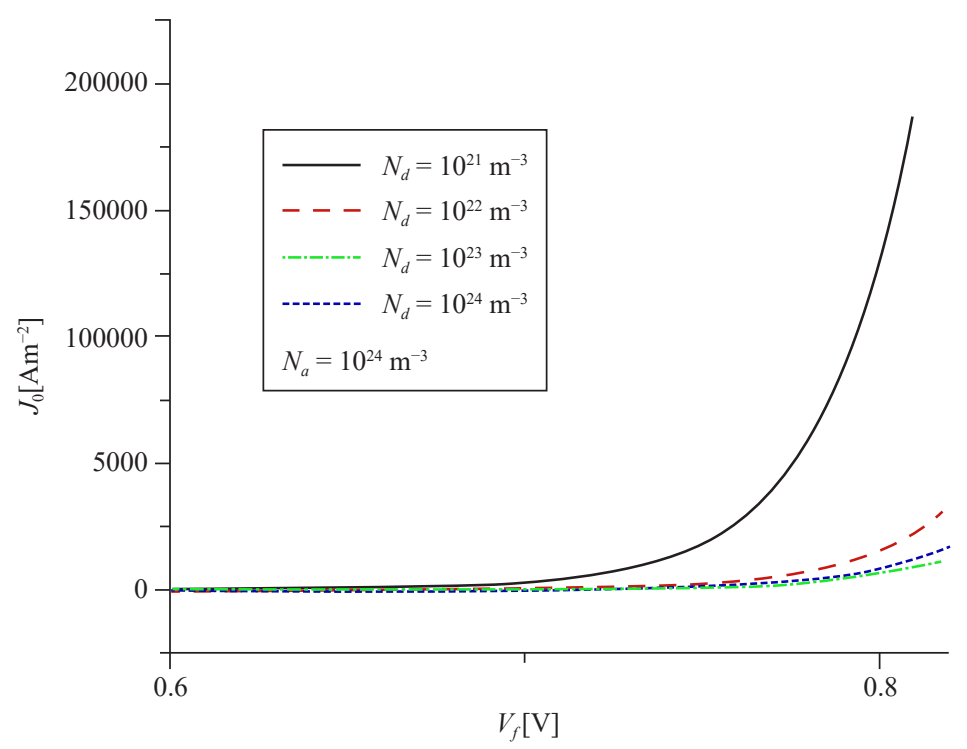

Figure 8: Dark current as a function of applied voltage at different doping concentrations. $N_{a}$ is fixed at $10^{24} \mathrm{~m}^{-3}$. 
Based on Figure 8, as doping concentration increases, the dark current (diode current) decreases. As doping increases (greater majority carrier concentration), the dark saturation current due to recombination decreases as it is inversely proportional to the doping concentration. ${ }^{21}$ This is because recombination is limited by the equilibrium minority concentration, thus a lower minority concentration would reduce the recombination rate. ${ }^{22}$ However, at $N_{d}=10^{24} \mathrm{~m}^{-3}$, the trend reverses due to the much lower $\tau_{h}$, which increases recombination. This explains the $V_{o c}$ and $F F$ profiles in Figures 4(b) and 4(c). Thus, maximising the photocurrent should not be the only goal as reducing the dark current contributes towards the increase in efficiency.

\section{CONCLUSION}

We have studied the doping-dependence of a nano-gap TPV device that uses a $p$-on- $n$ Si TPV cell. It is discovered that the highest conversion efficiency value is achieved when $N_{a}=10^{23} \mathrm{~m}^{-3}$ (lowest $N_{a}$ simulated) and $N_{d}=10^{22} \mathrm{~m}^{-3}$ while the greatest output power is generated when $N_{a}=10^{25} \mathrm{~m}^{-3}$ (highest $N_{a}$ simulated) and $N_{d}=10^{23} \mathrm{~m}^{-3}$. The optical response of the device depends very much on the doping concentration of the TPV cell. High doping levels would greatly increase free carrier absorption which causes greater radiative heat transfer between the radiator and the receiver especially at frequencies below the band gap. At high $N_{a}$ values, the optical response is less sensitive to the change in $N_{d}$. It is also discovered that the lowest donor concentration in the absorber region may not necessarily produce the best results despite the greater diffusion length. When the absorber thickness, $t_{a b s}$ is smaller than $L_{\text {eff } ;}$, the positive effects of a decreasing $D_{h}$ outweigh its negatives until $N_{d}$ is increased beyond an optimum value. This explains why $P_{m}$ is greatest when $N_{d}=10^{23} \mathrm{~m}^{-3}$. A decreasing diffusion coefficient is also partly the reason behind the increasing $P_{m}$ as $N_{a}$ is increased. Increasing the doping concentration also reduces the generation of dark current provided that the minority carrier lifetime is not too low. It is clear from the analysis done in this work that doping concentration has a heavy bearing on the performance of nanogap TPV devices and thus should be given great consideration when designing energy generators of this sort.

\section{ACKNOWLEDGEMENTS}

The authors would like to thank the Ministry of Science, Technology and Innovation of the Malaysian government for funding this project under the Science Fund (Project No. 06-02-14-SF0016). In addition, author J. Z-J. Lau is thankful to Swinburne University Sarawak, Malaysia for his postgraduate scholarship support. 


\section{REFERENCES}

1. Durán, J. C., Venier, G. \& Weht, R. (1991). Optimization of the junction depth and doping of solar cell emitters. Sol. Cells, 31(6), 497-503, https://doi.org/10.1016/0379-6787(91)90092-4.

2. Karazhanov, S. Z. (2000). Temperature and doping level dependence of solar cell performance including excitons. Sol. Energy Mater. Sol. Cells, 63(2), 149-163, https://doi.org/10.1016/S0927-0248(99)00173-7.

3. Ouyang, Z. et al. (2011). Influence of the absorber doping for p-type polycrystalline silicon thin-film solar cells on glass prepared by electron beam evaporation and solid-phase crystallization. J. Appl. Phys., 109(5), 054510, https://doi.org/10.1063/1.3553886.

4. Fu, C. \& Zhang, Z. (2006). Nanoscale radiation heat transfer for silicon at different doping levels. Int. J. Heat Mass Transfer, 49(9), 1703-1718, https://doi.org/10.1016/j.ijheatmasstransfer.2005.09.037.

5. Vaillon, R. et al. (2006). Modeling of coupled spectral radiation, thermal and carrier transport in a silicon photovoltaic cell. Int. J. Heat Mass Transf., 49(23), 4454-4468, https://doi.org/10.1016/j. ijheatmasstransfer.2006.05.014.

6. Mauk, M. \& Andreev, V. (2003). GaSb-related materials for TPV cells. Semicon. Sci. Technol., 18(5), S191.

7. Park, K. et al. (2008). Performance analysis of near-field thermophotovoltaic devices considering absorption distribution. J. Quant. Spectr. Radiat. Transf., 109(2), 305-316, https://doi.org/10.1016/j.jqsrt.2007.08.022.

8. Francoeur, M., Vaillon, R. \& Mengüç, M. P. (2011). Thermal impacts on the performance of nanoscale-gap thermophotovoltaic power generators. Energy Conv. IEEE Trans., 26(2), 686-698, https://doi.org/10.1109/ TEC.2011.2118212.

9. Francoeur, M., Mengüç, M. P. \& Vaillon, R. (2009). Solution of near-field thermal radiation in one-dimensional layered media using dyadic Green's functions and the scattering matrix method. J. Quant. Spectr. Radiat. Transf., 110(18), 2002-2018, https://doi.org/10.1016/j.jqsrt.2009.05.010.

10. Boriskina, S. V. et al. (2015). Enhancement and tunability of near-field radiative heat transfer mediated by surface plasmon polaritons in thin plasmonic films. Photonics, 2(2), 659-683, http://dx.doi.org/10.3390/ photonics2020659.

11. Yeh, P. (1988). Optical waves in layered media. New York: John Wiley \& Sons.

12. Green, M. A. (1998). Solar cells. Kensington: Prentice-Hall.

13. Patankar, S. (1980). Numerical heat transfer and fluid flow. New York: CRC Press. 
14. Palik, E. D. (1998). Handbook of optical constants of solids. London: Academic Press.

15. Collins, R. \& Fan, H. (1954). Infrared lattice absorption bands in germanium, silicon, and diamond. Phys. Rev., 93(4), 674, https://oi.org/10.1103/ PhysRev.93.674.

16. Kuźmicz, W. (1986). Ionization of impurities in silicon. Solid State Elect., 29(12), 1223-1227, https://doi.org/10.1016/0038-1101(86)90127-9.

17. Francoeur, M. (2010). Near-field radiative transfer: Thermal radiation, thermophotovoltaic power generation and optical characterization. Phd diss., University of Kentucky, United States.

18. Mulet, J. P. et al. (2002). Enhanced radiative heat transfer at nanometric distances. Microsc. Thermophys. Eng., 6(3), 209-222, https://doi. org/10.1080/10893950290053321.

19. Lau, J. Z. J., Bong, V. N. S. \& Wong, B. T. (2016). Parametric investigation of nano-gap thermophotovoltaic energy conversion. J. Quant. Spectr. Radiat. Transf., 171, 39-49, https://doi.org/10.1016/j.jqsrt.2015.11.023.

20. Streetman, B. G. \& Banerjee, S. (2000). Solid state electronic devices. New Jersey: Prentice Hall.

21. Luque, A. \& Hegedus, S. (2011). Handbook of photovoltaic science and engineering. New York: John Wiley \& Sons.

22. Van Zeghbroeck, B. (2004). Principles of semiconductor devices. Colorado: University of Colorado. 